\title{
Effect of Zn Substitution on the Magnetic and Magnetocapacitance Properties of Nanosized Multiferroic $\mathrm{GaFeO}_{3}$ Ceramics
}

\author{
Tai-Chun Han*, Chun Yen, Yi-De Chung, Meng-Lin Wu \\ Department of Applied Physics, National University of Kaohsiung, Taiwan \\ Email: *tchan@nuk.edu.tw
}

How to cite this paper: Han, T.-C., Yen, C., Chung, Y.-D. and Wu, M.-L. (2018) Effect of Zn Substitution on the Magnetic and Magnetocapacitance Properties of Nanosized Multiferroic $\mathrm{GaFeO}_{3}$ Ceramics. New Journal of Glass and Ceramics, 8 , 55-63.

https://doi.org/10.4236/njgc.2018.84005

Received: August 13, 2018

Accepted: October 8, 2018

Published: October 11, 2018

Copyright $\odot 2018$ by authors and Scientific Research Publishing Inc. This work is licensed under the Creative Commons Attribution International License (CC BY 4.0).

http://creativecommons.org/licenses/by/4.0/

cC) (i) Open Access

\begin{abstract}
This article aims to investigate the possibility to turn the multiferroic orders and magnetocapacitance effect close to/above room temperature in nanosized $\mathrm{GaFeO}_{3}$ ceramics by a sol-gel preparation method and substitution with non-magnetic $\mathrm{Zn}$ atoms. Therefore, in this work, we have synthesized a series of nanocrystalline $\mathrm{Ga}_{1-x} \mathrm{Zn}_{x} \mathrm{FeO}_{3}$ (GZFO, $x=0,0.01,0.05$ and 0.1 ) ceramic samples and study the effect of $\mathrm{Zn}$ substitution on their structural, magnetic, and electric properties. All the GZFO samples have an orthorhombic structure with $P C_{1} n$ space group and the value of lattice parameters increase systematically with increasing $\mathrm{Zn}$ concentration. Interestingly, it shows that magnetic and electric properties are strongly dependent on the $\mathrm{Zn}$ substitution concentration. Based on the results of temperature-dependent magnetizations, $\mathrm{M}(\mathrm{T})$, it is observed that with increasing $\mathrm{Zn}$-content up to 0.10 , the ferrimagnetic transition temperature $\left(\mathrm{T}_{\mathrm{C}}\right)$ increases from 306 to $320 \mathrm{~K}$. It is also found that the nanocrystalline $\mathrm{Zn}$-doped $\mathrm{GaFeO}_{3}(\mathrm{GFO})$ samples exhibit the characteristics of ferroelectricity at room temperature. Furthermore, the magnetization, ferroelectric polarization and magnetocapacitance of $\mathrm{Zn}$-doped GFO nanosized ceramics are enhanced compared to those of the pristine sample of GFO ferrite. These results open wide perspectives for the applications of room temperature multiferroic devices.
\end{abstract}

\section{Keywords}

Ferrite, Multiferroic, Ferrimagnetic, Ferroelectric, Ceramics, Zn Substitution

\section{Introduction}

Recently, multiferroic and magnetoelectric (ME) materials opened new avenues for the development of novel devices based on new functionalities such as con- 
trol of the magnetic properties by means of electrical fields and vice versa [1] [2]. Among these materials, gallium ferrite, $\mathrm{GaFeO}_{3}(\mathrm{GFO})$, has been reported to exhibit ferrimagnetic (FM) and piezoelectric orderings and has been intensively studied for its practical application as a ME ferrimagnet [3] [4]. GFO crystallizes in non-centrosymmetric orthorhombic structure with a space group $P C_{1} n$ and with four different cation sites, that is, labeled $\mathrm{Fe} 1$ and Fe2, which are predominantly occupied by $\mathrm{Fe}^{3+}$ and other two labeled as $\mathrm{Ga}$ and $\mathrm{Ga} 2$, which are mostly occupied by $\mathrm{Ga}^{3+}$ [4] [5]. The $\mathrm{Fe} 1, \mathrm{Fe} 2$, and Ga2 sites are octahedral surrounded by oxygen, while Gal site is tetrahedral coordinated by oxygen. In general, bulk GFO exhibits room temperature (RT) spontaneous polarization along the crystallographic $b$-axis and a FM structure below RT along the $c$-axis [4]. As such, GFO is a quite interesting material due to simultaneous exhibit magnetic and electric orderings [6]. In the past few years, several attempts have been directed towards the syntheses of new gallium ferrite compounds and studies of their related properties [7] [8]. Moreover, the substitution of trivalent or divalent metal ion in GFO is a pronounced way to control various physical properties [9] [10] [11]. However, the effect of the substitution of a non-magnetic cation on the net magnetization of a ferrimagnet is difficult to predict. It is depend on the site occupation of the non-magnetic cation with respect to the two opposing sublattices. The magnetization increase is possible, as in the case of the non-magnetic $\mathrm{Zn}^{2+}$-ions substitution into $\mathrm{NiFe}_{2} \mathrm{O}_{4}$ [12]. In addition, up to now, the $\mathrm{Zn}$ substitution effect on $\mathrm{T}_{\mathrm{C}}$ is not yet explored in literature. Accordingly, we aim to study the impact of substituting GFO with non-magnetic $\mathrm{Zn}^{2+}$ for $\mathrm{Ga}^{3+}$-ion on the multiferroism. In this work, therefore, we synthesized a series of nanocrystalline $\mathrm{Ga}_{1-x} \mathrm{Zn}_{x} \mathrm{FeO}_{3}$ (GZFO, $x=0,0.01,0.05$ and 0.1 ) ceramic samples by a sol-gel method to systematically study the effect of $\mathrm{Zn}$ substitution on their magnetic and multiferroic properties.

\section{Experimental Details}

\subsection{Sample Preparation}

The nanosized ceramic samples of $\mathrm{Ga}_{1-x} \mathrm{Zn}_{x} \mathrm{FeO}_{3}$ (GZFO, $x=0,0.01,0.05$ and 0.1 ) were prepared through a sol-gel method using nitrates as metal precursors. First, gallium nitrate $\left[\mathrm{Ga}\left(\mathrm{NO}_{3}\right)_{3} \cdot \mathrm{xH}_{2} \mathrm{O}\right]$, iron nitrate $\left[\mathrm{Fe}\left(\mathrm{NO}_{3}\right)_{3} \cdot 9 \mathrm{H}_{2} \mathrm{O}\right]$ and zinc nitrate $\left[\mathrm{Zn}\left(\mathrm{NO}_{3}\right)_{2} \cdot 6 \mathrm{H}_{2} \mathrm{O}\right]$ in stoichiometric proportions were dissolved in distilled water. Citric acid $\left(\mathrm{C}_{6} \mathrm{H}_{8} \mathrm{O}_{7}\right)$ in 1:1 molar ratio with respect to the metal nitrates was added to the solution as a complexant. The clear solution was dried at $120^{\circ} \mathrm{C}$ to form a gel, and then the obtain gel was burned until the combustion process was completed. After that, the precursory powders were reground and sintered at $800^{\circ} \mathrm{C}$ for $12 \mathrm{~h}$.

\subsection{Characterization}

The crystalline structure and the phase purity of the obtained samples were characterized with a typical X-ray diffraction (XRD), acquired by a Bruker D8 Ad- 
vance X-ray diffractometer $\mathrm{Cu} \mathrm{K}_{\alpha 1}$ radiation. The XRD data were fit using the General Structure Analysis System (GSAS) Rietveld refinement program. Micrographs of the samples were analyzed using a field emission transmission electron microscope (TEM) operated at $120 \mathrm{kV}$. The temperature- and field-dependent magnetizations were measured with a Quantum Design superconducting quantum interference device (SQUID) magnetometer. For the magnetocapacitance (MC) measurements, the powders were pressed into the disk $(5 \mathrm{~mm}$ in diameter and $0.5 \mathrm{~mm}$ in thickness) under a pressure of $1.5 \mathrm{GPa}$ and then coated with 100 $\mathrm{nm}$ thick silver layers on both the top and the bottom sides of the disk as electrodes. A capacitance bridge (Agilent E4980A Precision LCR meter) hooked to a probe station with a closed-cycle low temperature system was used. Room temperature ferroelectric measurements were performed using a Sawyer-Tower circuit and a commercial FE test system (TF Analyzer, aixACCT Co.).

\section{Results and Discussion}

\subsection{Crystal Structure}

The RT XRD patterns of the $\mathrm{Ga}_{1-x} \mathrm{Zn}_{x} \mathrm{FeO}_{3}$ samples with $x=0,0.01,0.05$, and 0.1 are given in Figure 1. Based on the standard reference (JCPDS Files No.26-0673), all the observed peaks of the GZFO samples can be indexed on the basis of an orthorhombic unit cell of space group, $P 2_{1} n$, suggesting that all samples without any secondary phase. The GZFO samples of various $\mathrm{Zn}$-content, namely, 0, 1, 5, and 10 at \% designated as GZFO1, GZFO2, GZFO3, and GZFO4, respectively. The lattice parameters were determined by Rietveld refinement method and listed in Table 1. As an example, the profile fitting of GZFO2 sample using GSAS Rietveld refinement program is shown in Figure 2. The fact that the value of the unit cell volume and the lattice parameters $a, b$, and $c$ of GZFO increase systematically with increasing $\mathrm{Zn}$-content $(x)$ implies that the $\mathrm{Zn}$-ions do replace the Ga-ions. This could be attributed to the larger ionic radius of $\mathrm{Zn}^{2+}$ ion $(\sim 0.74 \AA)$ than that of $\mathrm{Ga}^{3+}$ ion $(\sim 0.62 \AA)$, which clearly confirms that the zinc is successfully doped in the lattice of GFO.



Figure 1. XRD patterns of the $\mathrm{Ga}_{1-x} \mathrm{Zn}_{x} \mathrm{FeO}_{3}$ samples with $x=0,0.01,0.05$ and 0.1 . 
Table 1. Rietveld refinement data of GZFO samples with different Zn-content.

\begin{tabular}{ccccc}
\hline & GZFO1 & GZFO2 & GZFO3 & GZFO4 \\
\hline$a(\AA)$ & $8.7622(1)$ & $8.7645(3)$ & $8.7678(2)$ & $8.7715(4)$ \\
$b(\AA)$ & $9.4135(5)$ & $9.4164(2)$ & $9.4183(2)$ & $9.4216(1)$ \\
$c(\AA)$ & $5.0876(2)$ & $5.0901(5)$ & $5.0927(3)$ & $5.0955(1)$ \\
$c / a$ & 0.58063 & 0.58076 & 0.58084 & 0.58091 \\
$\sqrt{2} c l b$ & 0.76432 & 0.76446 & 0.76470 & 0.76485 \\
\hline
\end{tabular}



Figure 2. XRD patterns of the GZFO2 sample along with the Rietveld refinement. Inset shows the TEM image for the GZFO2 sample.

According to the calculation using a Debye-Scherrer equation [13], the average grain size of the GZFO samples are about $30 \mathrm{~nm}$. The grain sizes were also confirmed by TEM. As an example, the TEM image of the GZFO2 sample is shown in the inset of Figure 2. In order to measure the orthorhombic distortion [14], we have calculated the values of $c / a$ and $\sqrt{2} c / b$ of each GZFO samples (shown in Table 1). It is evident that the $\mathrm{Zn}$ substitution affects the structure of the pristine sample by increasing of the orthorhombic distortion of the perovskite lattice parameters of nanocrystalline GFO.

\subsection{Magnetic Properties}

The temperature dependence of magnetization, $M(T)$, was measured in a magnetic field of 1000 Oe under the condition of field-cooled (FC). Figure 3 displays the temperature-dependent FC magnetization for the GZFO samples with different $\mathrm{Zn}$ concentrations. The magnetic transition temperature $\left(\mathrm{T}_{\mathrm{C}}\right)$ is obtained for the GZFO samples as the differential minimum of the FC curves. The graphical representation of $d M / d T$ versus temperature curves gives the $T_{C}$ for the GZFO1 and GZFO4 samples, as shown in the inset of Figure 3. As can be seen, a typical FM to paramagnetic phase transition is observed for the GZFO1 sample, and the $T_{C}$ shifts to the higher temperatures and it equal to $320 \mathrm{~K}$ for the GZFO4 sample. The value of $\mathrm{T}_{\mathrm{C}}$ is rather high compare to the previously reported $\mathrm{T}_{\mathrm{C}}$ of 


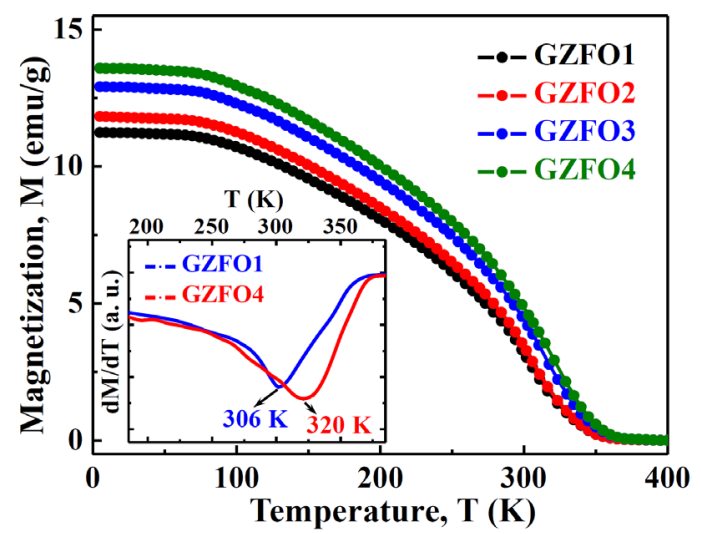

Figure 3. Temperature-dependent FC magnetization for the GZFO samples. Inset shows temperature dependence of the derivative of the FC curves for GZFO1 and GZFO4 samples.

$228 \mathrm{~K}$ in bulk GFO [4]. The results could be explained on the basis of variation of $\mathrm{Ga} / \mathrm{Fe}$ occupancy due to the disorder effects, which in turn alters the bond parameters of $\mathrm{Fe}-\mathrm{O}-\mathrm{Fe}$ and hence the strength of the magnetic interactions [4] [7].

The field-dependent magnetization $\mathrm{M}(\mathrm{H})$ curves of all the GZFO samples measured at $5 \mathrm{~K}$ are presented in Figure 4. And also, the $\mathrm{M}(\mathrm{H})$ loops for all the GZFO samples observed at RT are shown in the inset of Figure 4. On can observe from the $\mathrm{M}(\mathrm{H})$ data that all the samples exhibit pinched-like hysteresis loop behavior of two phase systems as indicated by the fact that the loops could be decomposed in two additive loops with different coercivities. A similar type of $\mathrm{M}-\mathrm{H}$ loop is reported by Kim et al. [5], in $\mathrm{GFO}$ and $\mathrm{AlFeO}_{3}$, and explained it in terms of the existence of hard and soft magnetic phases. Because of the fact that the four cation sites have different magnetic anisotropy energies in GFO, namely, the octahedral sites (F1, F2, and G2) have strong anisotropy, whereas the tetrahedral site (Ga1) is weak anisotropic, and hence these act as hard and soft magnetic phases, respectively [15]. In addition, the magnetization has been enhanced due to doping of $\mathrm{Zn}^{2+}$ in GFO compared to that of the pristine sample. The cause of magnetic enhancement could be explained from the modification exchange interaction due to the substitution of larger size cations of $\mathrm{Zn}^{2+}$ in place of relatively smaller size cations of $\mathrm{Ga}^{3+}$. Therefore, it is expected that with the variation of Zn-content in GZFO samples, there is a substantial change in cation distribution and hence in the magnetic properties.

\subsection{Electric Properties}

To investigate the presence of spontaneous polarization, the ferroelectric polarization-electric field (P-E) loops for the Zn-doped GFO samples were measured at RT are shown in Figure 5(a). Here, the P-E loops taken at an applied maximum electric field of $30 \mathrm{kV} / \mathrm{cm}$ for each samples. It is found that all three samples show clear hysteresis loop behaviors that confirms the existence of ferroelectricity. Figure 5(a) also shows that maximum polarization and the tendency 




Figure 4. Magnetic hysteresis loops $\mathrm{M}(\mathrm{H})$ measured at $5 \mathrm{~K}$ for all the GZFO samples. Inset shows $\mathrm{M}(\mathrm{H})$ loops of GZFO samples measured at RT.
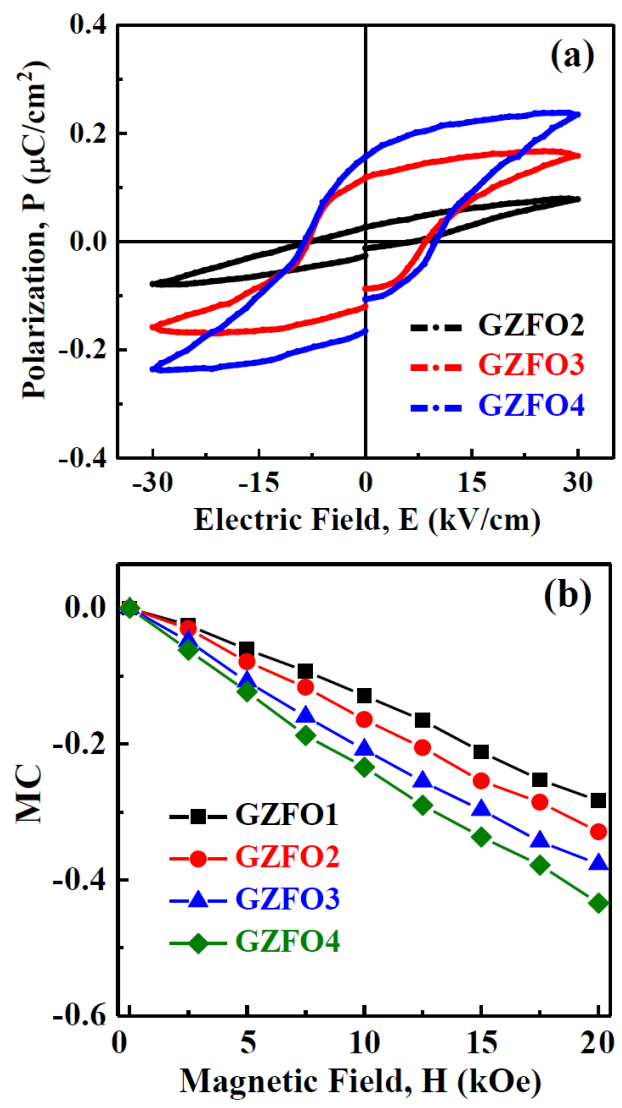

Figure 5. (a) P-E curves for GZFO2, GZFO3 and GZFO4 samples measured at RT with 1 $\mathrm{kHz}$ frequency; (b) The variation of MC with magnetic field for all the GZFO samples observed at RT.

of saturation increase with the increase of $\mathrm{Zn}$ concentration. From the P-E loops, we see that the values of the maximum polarization of GZFO2, GZFO3 and GZFO4 samples are $0.08,0.16$ and $0.23 \mu \mathrm{C} / \mathrm{cm}^{2}$, respectively. The enhancement of electric polarization may be ascribed to the increase in distortion of the crystal lattice due to the substitution of $\mathrm{Zn}^{2+}$-cations in place of $\mathrm{Ga}^{3+}$-cations in the 
doped samples.

In order to know the coupling between the magnetic and ferroelectric orderings, we have measured the dielectric constant of the GZFO samples in presence of an externally applied magnetic field. The change in dielectric constant with the applied magnetic field is one of the important ways to study the coupling effect [16] [17]. Figure 5(b) shows the variation of MC which is calculated by the expression [17], MC $=\left[\varepsilon^{\prime}(\mathrm{H}, \mathrm{T})-\varepsilon^{\prime}(0, \mathrm{~T})\right] / \mathcal{\varepsilon}^{\prime}(0, \mathrm{~T})$. Here, $\varepsilon^{\prime}(\mathrm{H}, \mathrm{T})$ and $\varepsilon^{\prime}(0, \mathrm{~T})$ represents the dielectric constants in presence and absence of external magnetic field $(\mathrm{H})$, respectively. In such GZFO sample, the dielectric constant is strongly perturbed by the external magnetic field. It is confirmed the presence of FM and ferroelectric coupling, that is, the multiferroic state in all the GZFO samples at $\mathrm{RT}$. It is to be noted that the value of MC increases in the doped samples for different applied magnetic fields. And also, the values of MC for nanosized GZFO ceramic samples are higher than that observed for bulk GFO [6]. To further probe the electrical leakage effect, the leakage current densities $(J)$ were measured for all the GZFO samples at RT as shown in Figure 6. It shows that the Zn substitution in GFO allows decreasing the leakage current density by two orders of magnitude, as shown in the inset of Figure 6. We attribute the decrease of the leakage currents to the substitution of $\mathrm{Fe}^{2+}$ with $\mathrm{Zn}^{2+}$, decreasing the hopping possibilities between $\mathrm{Fe}^{2+}$ and $\mathrm{Fe}^{3+}$ within the structure; a similar behavior has been reported by Lefevre et al. [18].

\section{Conclusion}

In summary, we have studied the effect of $\mathrm{Zn}$ doping on the multiferroic properties of nanocrystalline GZFO samples prepared by a sol-gel method. It shows that magnetic and electric properties are strongly dependent on the $\mathrm{Zn}$-doping. The magnetic characterization indicates that with an increase in the $\mathrm{Zn}$-content, the FM transition temperature increases from 306 to $320 \mathrm{~K}$. It is also found that

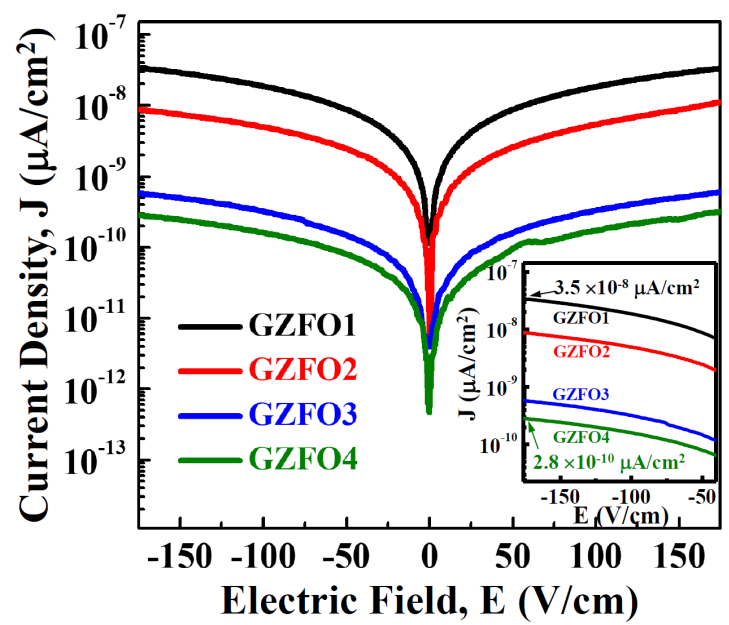

Figure 6. Leakage current density (J) as function of applied electric field (E) measured at RT for the GZFO samples. Inset shows an enlarged view of the raw data for the GZFO samples at negative electric field. 
the Zn-doped GFO samples exhibit the characteristics of ferroelectricity at RT. Based on the overall data, one can suggest that the $\mathrm{Zn}$-substitution in GFO introduces structural distortion and modifies magnetic exchange interaction, which affects both FM and ferroelectric orderings and MC effect. Furthermore, the simultaneous enhancement of magnetization and electric polarization together with the enhanced values of MC in $\mathrm{Zn}$-doped samples are prominent candidates for the applications of multiferroic devices.

\section{Acknowledgements}

This research was financially supported by Ministry of Science and Technology of Taiwan, under Grant no. MOST-107-2112-M-390-003 and 105-2112-M-390-001.

\section{Conflicts of Interest}

The authors declare no conflicts of interest regarding the publication of this paper.

\section{References}

[1] Khomskii, D. (2009) Trend: Classifying Multiferroics: Mechanisms and Effects. Physics, 2, 20. https://doi.org/10.1103/Physics.2.20

[2] Wang, K.F., Liu, J.M. and Ren, Z.F. (2009) Multiferroicity: The Coupling between Magnetic and Polarization Orders. Advances in Physics, 58, 321-448. https://doi.org/10.1080/00018730902920554

[3] Rado, G.T. (1964) Observation and Possible Mechanisms of Magnetoelectric Effects in a Ferromagnet. Physical Review Letters, 13, 335. https://doi.org/10.1103/PhysRevLett.13.335

[4] Arima, T., Higashiyama, D., Kaneko, Y., He, J.P., Goto, T., Miyasaka, S., Kimura, T., Oikawa, K., Kamiyama, T., Kumai, R. and Tokura, Y. (2004) Structural and Magnetoelectric Properties of $\mathrm{Ga}_{2-\mathrm{x}} \mathrm{Fe}_{\mathrm{x}} \mathrm{O}_{3}$ Single Crystals Grown by a Floating-Zone Method. Physical Review B, 70, 064426.

https://doi.org/10.1103/PhysRevB.70.064426

[5] Han, M.J., Ozaki, T. and Yu, J. (2007) Magnetic Ordering and Exchange Interactions in Multiferroic $\mathrm{GaFeO}_{3}$. Physical Review B, 75, 060404(R). https://doi.org/10.1103/PhysRevB.75.060404

[6] Naik, V.B. and Mahendiran, R. (2009) Electrical, Magnetic, Magnetodielectric, and Magnetoabsorption Studies in Multiferroic $\mathrm{GaFeO}_{3}$. Journal of Applied Physics, 106, 123910. https://doi.org/10.1063/1.3271391

[7] Mohamed, M.B., Senyshyn, A., Ehrenberg, H. and Fuess, H. (2010) Structural, Magnetic, Dielectric Propertiesof Multiferroic $\mathrm{GaFeO}_{3}$ Prepared by Solid State Reaction and Sol-Gel Methods. Journal of Alloys and Compounds, 492, L20-L27. https://doi.org/10.1016/j.jallcom.2009.11.099

[8] Mukherjee, S., Ranjan, V., Gupta, R. and Garg, A. (2012) Compositional Dependence of Structural Parameters, Polyhedral Distortion and Magnetic Properties of Gallium Ferrite. Solid State Communications, 152, 1181-1185. https://doi.org/10.1016/j.ssc.2012.03.010

[9] Mohamed, M.B. and Fuess, H. (2011) Effect of Mn Doping on Structural and Magnetic Properties of $\mathrm{GaFeO}_{3}$. Journal of Magnetism and Magnetic Materials, 323, 
2090-2094. https://doi.org/10.1016/j.jmmm.2011.03.019

[10] Han, T.C., Lee, Y.C. and Chu, YT. (2014) Effect of Cobalt Doping on Site-Disorder and Magnetic Behavior of Magnetoelectric $\mathrm{GaFeO}_{3}$ Nanoparticles. Applied Physics Letters, 105, 212407. https://doi.org/10.1063/1.4902874

[11] Thomasson, A., Cherifi, S., Lefevre, C., Roulland, F., Gautier, B., Albertini, D., Meny, C. and Viart, N. (2013) Room Temperature Multiferroicity in $\mathrm{Ga}_{0.6} \mathrm{Fe}_{1.4} \mathrm{O}_{3}$ : MgThin Films. Journal of Applied Physics, 113, 214101. https://doi.org/10.1063/1.4808349

[12] Akther Hossain, A.K.M., Mahmud, S.T., Seki, M., Kawai, T. and Tabata, H. (2007) Structural, Electrical Transport, and Magnetic Properties of $\mathrm{Ni}_{1-\mathrm{x}} \mathrm{Zn}_{\mathrm{x}} \mathrm{Fe}_{2} \mathrm{O}_{4}$. Journal of Magnetism and Magnetic Materials, 312, 210-219. https://doi.org/10.1016/j.jmmm.2006.09.030

[13] Patterson, A.L. (1939) The Scherrer Formula for X-Ray Particle Size Determination. Physical Review, 56, 978. https://doi.org/10.1103/PhysRev.56.978

[14] Paszkowicz, W., Piętosa, J., Woodley, S.M., Dłużewski, P.A., Kozłowski, M. and Martin, C. (2010) Lattice Parameters and Orthorhombic Distortion of $\mathrm{CaMnO}_{3}$. Powder Diffraction, 25, 46-59. https://doi.org/10.1154/1.3314256

[15] Kim, W., We, J.H., Kim, S.J. and Kim, C.S. (2007) Effects of Cation Distribution for $\mathrm{AFeO}_{3}(\mathrm{~A}=\mathrm{Ga}, \mathrm{Al})$. Journal of Applied Physics, 101, 09M515.

[16] Sun, Z.H., Cheng, B.L., Dai, S., Cao, L.Z., Zhou, Y.L., Jin, K.J., Chen, Z.H. and Yang, G.Z. (2006) Dielectric Properties Studies of Multiferroic $\mathrm{GaFeO}_{3}$. Journal of Physics D: Applied Physics, 39, 2481-2484. https://doi.org/10.1088/0022-3727/39/12/001

[17] Li, M., Ning, M., Ma, Y., Wu, Q. and Ong, C.K. (2007) Room Temperature Ferroelectric, Ferromagnetic and Magnetoelectric Properties of Ba-Doped $\mathrm{BiFeO}_{3}$ Thin Films. Journal of Physics D: Applied Physics, 40, 1603-1607. https://doi.org/10.1088/0022-3727/40/6/002

[18] Lefevre, C., Shin, R.H., Lee, J.H., Oh, S.H., Roulland, F., Thomasson, A., Autissier, E., Meny, C. Jo, W. and Viart, N. (2012) Reduced Leakage Currents and Possible Change Carriers Tuning in Mg-Doped $\mathrm{Ga}_{0.6} \mathrm{Fe}_{1.4} \mathrm{O}_{3}$ Thin Films. Applied Physics Letters, 100, 262904. https://doi.org/10.1063/1.4729872 